\title{
Modelling of an Upflow Anaerobic Sludge Blanket reactor
}

\author{
R. Rodriguez ${ }^{1,2}$ \& L. Moreno ${ }^{2}$ \\ ${ }^{I}$ Universidad Nacional de Ingeniería (UNI), Nicaragua \\ ${ }^{2}$ Royal Institute of Technology, Sweden
}

\begin{abstract}
A model describing both physical and biological processes in Upflow Anaerobic Sludge Blanket (UASB) reactors was developed. The main objective of the modelling was to take into account the transient growth of the microorganisms from the start-up of the reactor until a steady state is reached. In addition, the model considers the degradation of the substrate and its reaction with the biomass, which is present in the form of spherical granules of different sizes. For the degradation of the substrate within the granule, the mass transport through the stagnant film around the granule and the intra-particle diffusion are accounted for, together with the specific reaction rate.

In the model, the growth of the biomass follows the Contois kinetics. The amount of biomass reaches a steady state after weeks or months. Biomass is generated when substrate is degraded and a given fraction of biomass disappears per unit of time, which is determined by the decay constant. The value of this constant is taken from the literature. The model also considers that a fraction of the biomass may be carried out by the water flow.

The model was solved by using COMSOL Multiphysics. Data from the literature was used in order to illustrate the processes occurring in the UASB reactor. Modelling can be a useful tool for the design and optimization of UASB reactors.
\end{abstract}

Keywords: UASB, COMSOL multiphysics, Contois model.

\section{Introduction}

Biological anaerobic treatment is extensively used to treat wastewater containing high levels of organic matter. Many environmental studies focus on the 
degradation of wastewater in anaerobic reactors due to the fact that this type of processes not only degrades the organic matter, but also produces fuel in the form of biogas and generates low quantities of sludge, making the reactor's maintenance less frequent. One of these reactors is the Upflow Anaerobic Sludge Blanket (UASB) reactor.

The Contois model (Contois [1]) describes the growth of microorganisms with time. This model was developed about 20 years after the Monod model. The principal difference between these two models is that the Monod model considers that the concentration of limiting substrate (when the specific growth rate is half maximum, commonly written as $K_{S}$ ) is independent of population density, whereas the Contois model says that $K_{S}$ is a function of population density (Contois [1]). Several researchers have compared the Contois vs. the Monod models. However, they have not reached any clear conclusion about which model is better. Bhattacharya and Khai [2] report that the Contois model is better for modelling cow dung digesters. According to Kryłów and Tal-Figiel [3], in order to describe the kinetics of individual stages of processes (substrate degradation, VFA behaviour, microbial growth) for low strength municipal wastewater, it is more convenient to use the Monod model. Hu et al. [4] studied the kinetics of anaerobic digestion using synthetic ice-cream wastewater and they reported that the Contois equation is more adequate than the Monod equation for describing the kinetics process.

Despite the fact that UASB reactors have been used for more than 20 years, few models describing the processes exist. The main processes occurring in the UASB reactors are degradation of substrate, microorganism growth and production of biogas. Singhal et al. [5] developed a model describing the fluid flow of UASB reactors; they divided the reactor in two zones (volume zone and bypassing zone) instead of the three typical zones (sludge bed, sludge blanket and settling zones) to reduce the amount of variables of the model. They injected lithium tracer in the UASB reactor influent and monitored it in the reactor's effluent at different times of the reactor operation. They concluded that the model is suitable for explaining the physical transitions occurring in the zones of UASB reactors. Kalyuzhnyi et al. [6] developed a model focusing on sludge dynamics along the reactor height. The model is one-dimensional dispersed plug flow. It was tested using literature data and as a result, Kalyuzhnyi et al. [6] reported that the model sometimes overestimated or underestimated data from literature in accordance with the working conditions of the model. Korsak et al. [7] developed a model describing processes occurring in UASB reactors. They included the kinetics reactions based on the Monod model.

In this paper, a model describing both physical and biological processes in an Upflow Anaerobic Sludge Blanket (UASB) reactor is developed. The model takes into account the transient growth of the microorganisms and substrate degradation from the start-up of the reactor until a steady state is reached. In addition, the model considers the degradation of the substrate within the biomass, which is present in the form of spherical granules of different sizes. In the granule, the mass transport through the stagnant film around the granule and 
the intra-particle diffusion are considered accounted for, together with the specific reaction rate. It is assumed that the kinetics follow the Contois model.

The modelling of UASB reactors processes could be a helpful tool for designing and monitoring reactors used in treatment plants.

\section{The model}

The proposed model explains the substrate behaviour and microorganism growth along the reactor height. The model is one dimensional (all processes depend on the vertical axis). The model kinetics follow the Contois model (equation (1)),

$$
\mu=\mu_{\max } \frac{S}{B \cdot X+S}
$$

where $\mu$ is the specific growth rate $\left(\mathrm{h}^{-1}\right), \mu_{\max }$ is the maximum specific growth rate $\left(\mathrm{h}^{-1}\right), S$ is the substrate concentration $\left(\mathrm{Kg} / \mathrm{m}^{3}\right), B$ is the kinetic parameter [KgCOD $\left(\mathrm{KgVSS}^{-1}\right]$ and $X$ is microorganism concentration $\left(\mathrm{Kg} / \mathrm{m}^{3}\right)$.

The model also includes the decay rate of microorganisms that could be caused by toxic metabolism (Kryłów and Tal-Figiel [3]), breaking of the cellular membrane (Kalyuzhnyi et al. [8]) and predation (Bitton [9]). The model takes into account the fraction of microorganisms (biomass) present in the reactor effluent. García et al. [10] studied two identical UASB reactors. They focused on the wash out fraction. They added polymers to one of the reactors and reported that this addition improved the degree of biomass retention inside the reactor.

In order to model the main processes occurring in the UASB reactor, some simplifications or assumptions are made:

-The processes depend on the height of the reactor (one dimensional model)

-Substrate is comprised of a single biodegradable substrate.

-The particles or granules have a spherical shape.

-There is no temperature change inside the reactor.

-Kinetics follows the Contois model

Applying the assumptions described above, the differential equation describing the concentration of substrate in the reactor may be written as

$$
\begin{gathered}
\frac{\partial S}{\partial t}=D \frac{\partial^{2} S}{\partial Z^{2}}-v e l \frac{\partial S}{\partial Z}-\left(\frac{\mu_{\max }}{Y} \frac{X}{B \cdot X+S}\right) \cdot S \\
\frac{\partial X}{\partial t}=\left(D^{*} w\right) \frac{\partial^{2} X}{\partial Z^{2}}-\left[\left(v^{*} l^{*} w\right)\right] \frac{\partial X}{\partial Z}+\left(\mu_{\max } \frac{S}{B \cdot X+S}-K_{d}\right) \cdot X
\end{gathered}
$$

where $S$ is the substrate concentration $\left[\mathrm{Kg} \mathrm{m}^{-3}\right], X$ is the biomass concentration $\left[\mathrm{Kg} \mathrm{m}^{-3}\right], D$ is the dispersion coefficient $\left[\mathrm{m}^{2} \mathrm{~h}^{-1}\right]$, vel is the upflow velocity $[\mathrm{m}$ $\left.\mathrm{h}^{-1}\right], Y$ is the yield coefficient $\left[\mathrm{KgVSS}(\mathrm{KgCOD})^{-1}\right], w$ is the washout fraction 
which represents a percentage of microorganisms present in the effluent, $t$ is time $[\mathrm{h}]$ and $Z$ is the vertical axis, refers to the height of the reactor.

In both equations (2) and (3) the first term on the left hand side is the accumulation term, the first term on the right hand side is the dispersion term, the second one is the advective or transport term and the third one is the reaction term.

In a UASB reactor, the microorganisms are found in the reactor forming granules or particles. The reaction takes place mainly within the particles and depending on the reaction rate, they grow with time. The growth rate is high in zones with high activity and substrate degradation and small in the region with low activity.

The location of the granules in the reactor is determined mainly by the size of the granules and the up-flow velocity of the water; i.e. by the action of the gravity and the friction forces particle-fluid. Gas generation is also very important, since the gas bubbles adhere to the particles and lift them. The location of the particles is continuously varying, therefore only an average location along the vertical axis may be defined for the particles in function of the size; large size granules are found in locations close to the bottom.

\subsection{The reaction within the granule}

For the interaction between substrate degradation, microorganism growth and particle (granule) size, a model was developed which allows determining the kinetics constant defined in equations (7) and (8).

In the model, it is assumed that the reaction of degradation of the substrate takes place within the granules, in presence of microorganisms. The model includes the transport of substrate by diffusion through the stagnant water film around the granules, the diffusion from the surface into the granule where the degradation reaction takes place, and finally the degradation reaction. In summary, we can talk about three resistances in series. Due to the biological reaction, microorganisms grow and the granule is increased in size. Since the variations in substrate concentration within the granules are small with time, a quasi steady state is obtained for the concentration of substrate within the granule. Therefore, the mass balance for the substrate in the granule may be written for the steady state.

$$
\frac{1}{r^{2}} \frac{d}{d r}\left(r^{2} \frac{d S_{p}}{d r}\right)=\frac{k}{D_{A}} S_{p}
$$

where $D_{A}$ is the effective diffusion coefficient of substrate within the granule $\left[\mathrm{m}^{2} \mathrm{~h}^{-1}\right], S_{p}$ is the concentration of substrate within the granule (particle), $\left[\mathrm{kgm}^{-3}\right] ; \mathrm{k}$ is the substrate volumetric conversion rate, $\left[\mathrm{h}^{-1}\right]$; and $\mathrm{r}$ is the radial distance from the centre of the particle, $[\mathrm{m}]$. The equation is solved for a substrate concentration $S\left[\mathrm{kgm}^{-3}\right]$ in the bulk liquid, and for a mass transfer coefficient $k_{m}\left[\mathrm{~m} \mathrm{~h}^{-1}\right]$ for the substrate through the stagnant water film around 
the granule. Once the concentration profile for the substrate in the granule is known, the mass flow of substrate into the particle, $W_{R}\left[\mathrm{kgh}^{-1}\right]$, may be obtained [5]:

$$
W_{R}=4 \pi R^{2} k_{m} S \cdot\left(\frac{D_{A}(\phi \cosh \phi-\sinh \phi)}{D_{A}(\phi \cosh \phi-\sinh \phi)+R k_{m} \sinh \phi}\right)
$$

where $\phi=R \sqrt{k / D_{A}}$ is the Thiele modulus (dimensionless value) and $R$ is the granule radius [m]. The term $W_{R}$ corresponds to the amount of substrate that is degraded within each granule. Therefore, the amount of substrate degraded per unit volume reactor may be calculated considering $\varphi_{P}$, which is the fraction of total volume occupied by the granules $\left[\mathrm{m}^{3}\right.$ granule $\left(\mathrm{m}^{3}\right.$ reactor) $\left.{ }^{-1}\right]$. Once the substrate degradation rate is known, the kinetics constant $(\mathrm{K})$ in equations (7) and (8) may be determined,

$$
K=3 k_{m} \frac{\varphi_{p}}{R}\left[\frac{D_{A}(\phi \cosh \phi-\sinh \phi)}{D_{A}(\phi \cosh \phi-\sinh \phi)+R k_{m} \sinh \phi}\right]
$$

The model for the substrate and microorganisms concentration in the UASB reactor is transient and the location of the granules along of the reactor height is determined by their size. However, the reaction within the granules is solved for the steady state. The governing equation for substrate degradation and microorganism growth may be rewritten in function of the kinetic constant and they are shown in equations (7) and (8), respectively.

$$
\begin{gathered}
\frac{\partial S}{\partial t}=D \frac{\partial^{2} S}{\partial Z^{2}}-v e l \frac{\partial S}{\partial Z}-(K \cdot S) \\
\frac{\partial X}{\partial t}=\left(D^{*} w\right) \frac{\partial^{2} X}{\partial Z^{2}}-[(v e l * w)] \frac{\partial X}{\partial Z}+\left(K \cdot Y \cdot S-K_{d} \cdot X\right)
\end{gathered}
$$

In equation (8) the decay constant $\left(K_{d}\right)$ is included. This constant represents the microorganism death and its unit is $\left[\mathrm{h}^{-1}\right]$. The parameter $w$ in equation (8) represents the fraction of microorganisms that wash out by the upflowing water. It is important to indicate that the amount of biomass is included in the Kinetics constant through the value of $\varphi_{P}$.

\subsection{Model data}

Sponza and Uluköy [11] compared three models (Monod, Modified StoverKincannon and Grau second order) in order to determine which one is suitable 
for predicting the substrate removal kinetics of a UASB reactor. They used synthetic wastewater containing glucose and 2,4 dichlorophenol (2,4 DCP) as substrate for feeding the reactor. As a result, Sponza and Uluköy reported Monod kinetic model was more suitable than Stover-Kincannon and Grau second order to describe the reactor's behaviour. In this paper we used data reported by Sponza and Uluköy [11], but we applied Contois model in place of Monod model to test our model. Table 1 shows the parameters used in the model. It is assumed that the substrate concentration both in input flow and initial is the same $\left(3 \mathrm{Kg} / \mathrm{m}^{3}\right)$. However, in the input flow, there is no microorganism concentration but the initial microorganism concentration in the UASB reactor is $5 \mathrm{E}-3 \mathrm{Kg} / \mathrm{m}^{3}$. The kinetic parameter $\mathrm{B}$ was determined plotting the $\mathrm{SRT} /\left(1+\mathrm{SRT}^{*} \mathrm{Kd}\right)$ vs. $\mathrm{X} / \mathrm{S}$ where SRT is the sludge retention time (Sponza and Işik [12]).

The experimental UASB reactor has $1 \mathrm{~m}$ of length and a diameter of $6 \mathrm{~cm}$, the effective volume is 2.5 litres and the UASB reactor was inoculated with partial granules collected from a UASB reactor treating wastewater of Pakmaya Baker Factory in Izmir but the input fluid flow for the experiments was synthetic wastewater. The reactor worked under mesophilic conditions (Sponza and Uluköy [11]).

Both microorganism and substrate performance was set for a period of 648 hours (HRT varying 20-10 h), by Sponza and Uluköy [11]. To know the behaviour of substrate, chemical oxygen demand (COD) data was used as substrate. The washout fraction was assumed as well as the Péclet number. In Figures 1 and 2, the $\mathrm{x}$-axis represents the reactor height $(\mathrm{m})$ and the $\mathrm{y}$-axis is the substrate and microorganism concentration $\left(\mathrm{Kg} / \mathrm{m}^{3}\right)$ respectively. Figure 3 , the $\mathrm{x}$ axis is the reactor's height $(\mathrm{m})$ and the $y$-axis is the granule's radius $(\mathrm{m})$.

Table 1: $\quad$ Parameters used in the model.

\begin{tabular}{|c|c|c|}
\hline Parameter & Value & Units \\
\hline Péclet number & 50 & Dimensionless \\
\hline Hydraulic retention time* & 9 & $\mathrm{~h}$ \\
\hline Upflow velocity* & 0.098 & $\mathrm{~m}^{3} / \mathrm{m}^{2} \cdot \mathrm{h}$ \\
\hline Dispersion coefficient & $1.96 \mathrm{E}-3$ & $m^{2} h^{-1}$ \\
\hline $\begin{array}{l}\text { Maximum specific growth } \\
\text { rate* }\end{array}$ & $8.75 \mathrm{E}-3$ & $\mathrm{~h}^{-1}$ \\
\hline Yield* & 0.00078 & $\mathrm{KgVSS}(\mathrm{KgCOD})^{-1}$ \\
\hline Kinetic parameter $\mathrm{B}^{*}$ & 0.1053 & $\mathrm{KgCOD}(\mathrm{KgVSS})^{-1}$ \\
\hline Decay constant* & $3.875 \mathrm{E}-5$ & $h^{-1}$ \\
\hline Washout fraction & 0.0002 & Fraction microorganism \\
\hline
\end{tabular}

* Taken from Sponza and Uluköy [11]. 


\section{Results and discussion}

The equations of the model were solved using COMSOL Multiphysics 3.5. The model response for substrate degradation and microorganism growth are shown in Figures 1 and 2. Figure 3 shows the radius of the granule as a function of the height in the reactor.

Regarding the substrate behaviour, the input substrate concentration is 3 $\mathrm{Kg} / \mathrm{m}^{3}$, but due to dispersion, Figure 1 shows a value slightly less $\left(2.9 \mathrm{Kg} / \mathrm{m}^{3}\right)$. The substrate concentration decreases continuously with the height, reaching a value below $1.5 \mathrm{Kg} / \mathrm{m}^{3}$ at the reactor top. The steady state is reached after a long time of operation (more than $650 \mathrm{~h}$ ).

In Figure 2, the microorganism concentration reaches its maximum at the reactor bottom, where the maximum substrate degradation rate takes place. The concentration of microorganisms decreases with the height due to the less concentration of substrate. An increase of biomass concentration at the reactor bottom is remarkable compared to that of other heights of the reactor. The behaviour of the reactor is very sensible to the parameters chosen in the simulations. In this case, the concentration of the microorganisms in the reactor is lower for the combination of parameters used.

Sponza and Uluköy [11] reported a COD (substrate) reduction of $83 \%$ with an operation time of 27 days. In our model, the COD is consumed at the reactor's bottom where the major concentration of biomass is present. With respect to Figure 2, Sponza and Uluköy did not report values of the microorganism

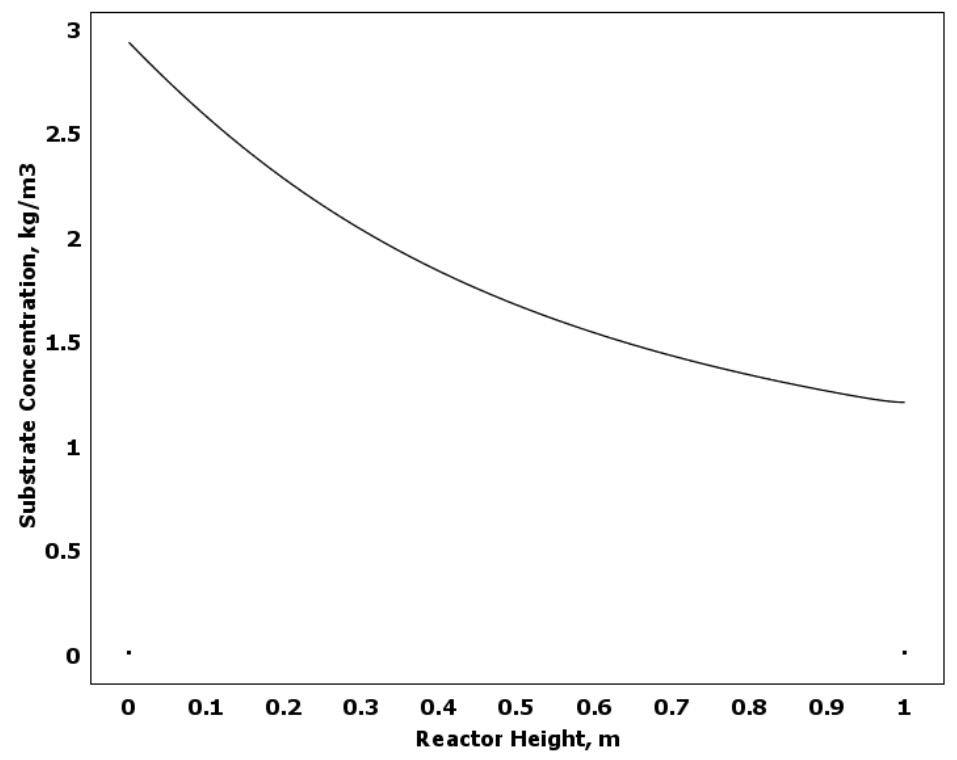

Figure 1: $\quad$ Model response for the substrate concentration profile. 
308 Water Pollution X

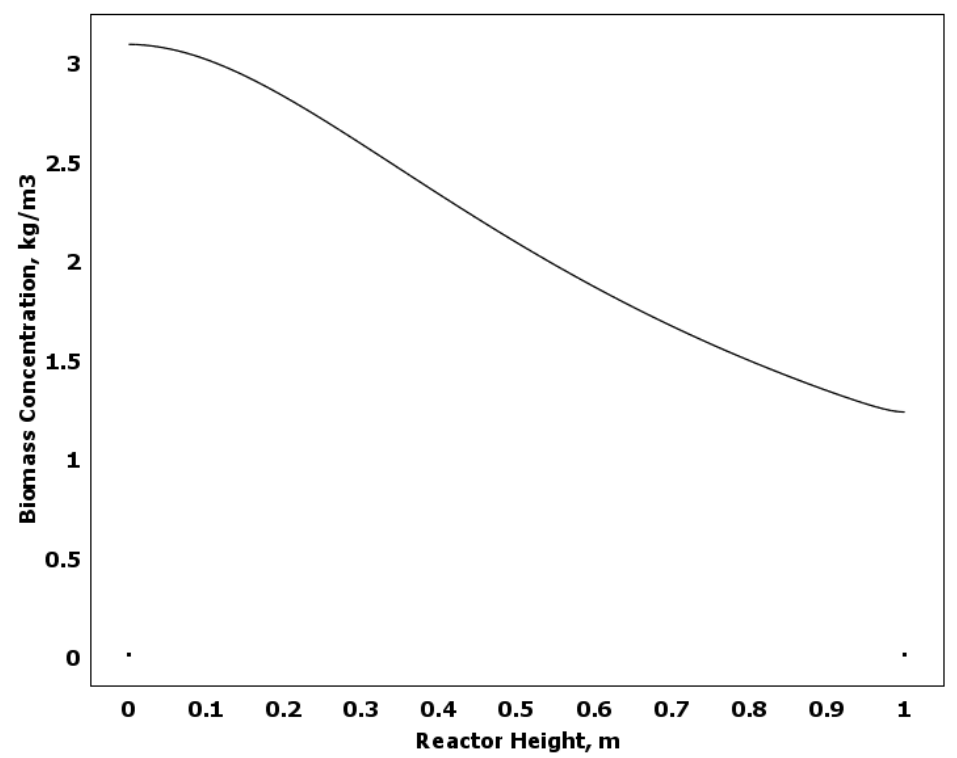

Figure 2: $\quad$ Model response for the microorganism concentration profile.

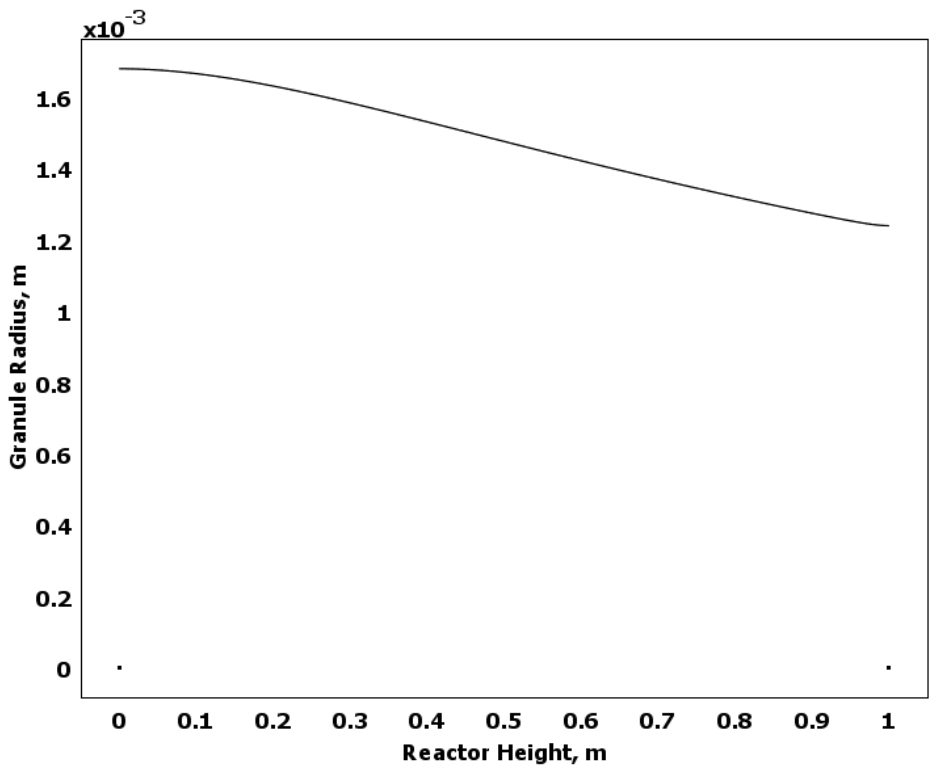

Figure 3: $\quad$ Model response for the granule radius. 
behaviour; their work was focused on the comparison of three kinetics models. Initial biomass concentration was $5 \mathrm{E}-3 \mathrm{Kg} / \mathrm{m}^{3}$, but after long operation time, biomass grows and reaches $3.53 \mathrm{Kg} / \mathrm{m}^{3}$. The major growing of biomass is found at the reactor's bottom.

Figure 3 shows the profile of granules sizes along the reactor's height. The response model shows granule diameters ranging between $2.5-3.2 \mathrm{~mm}$, after a long operation time.

In our model, we have it transient but with time it reaches steady state conditions. An important aspect in this modelling is the high sensibility of the model response to the value used for the different parameters. A study of the influence of the most important parameters on the response of the model is currently in process.

\section{Conclusion}

The proposed model describes the degradation of the substrate (as COD) and the growth of the microorganisms (biomass) in the reactor. The model has been successfully used to simulate the UASB reactor performance studied by Sponza and Uluköy [11]. Two kinetics models, Contois and Monod were used to study the results and no significant differences were found between these kinetics. These kinetics reactions include the growth and death of the microorganisms. Since the reaction takes place within the granules a model was developed which takes into account diffusion resistance in the granule and in the film around the granule. The granules in the reactor are distributed according their sizes, the large ones trend to locate at the bottom of the reactor.

The results of the model are quite sensitive to some model parameters used in the simulations; e.g. the relative values between the decay constant, $\mathrm{K}_{\mathrm{d}}$, and the growth rate. If the decay constant is too large, the microorganisms will decrease in the reactor, decreasing the degradation of the substrate.

This type of model may be a useful tool in the development and design of UASB reactors. It allows study the consequences on the reactor performance of different types of substrates, inlet substrate concentrations, flowrates, and different kind of biomasses.

\section{Acknowledgement}

This work is supported by the Swedish International Development Cooperation Agency (SIDA/SAREC).

\section{References}

[1] Contois D. Kinetics of Bacterial Growth: Relationship between Population Density and Specific Growth Rate of Continuous Cultures. Microbial. 21, 40-50, 1959. 
[2] Bhattacharya S. and Khai P. Kinetics of Anaerobic Cowdung Digestion. Energy Vol. 12, No. 6, pp. 497-500, 1987.

[3] Kryłów M. and Tal-Figiel B. Kinetics of Anaerobic Processes. Proceedings of a Polish-Swedish seminar, 2003.

[4] Hu W., Thayanithy K. and Forster C. A Kinetic Study of the Anaerobic Digestion of Ice-cream Wastewater. Process Biochemistry 37, 965-971, 2002.

[5] Singhal A., James Gomes, Praveen V. and Ramachandran B. Axial Dispersion Model for Upflow Anaerobic Sludge Blanket Reactors. Biotechnol. Prog. 14, 645-648, 1998.

[6] Kalyuzhnyi S. Fedorovich V. and Lens P. Novel Dispersed Plug Flow Model for UASB Reactors Focusing on Sludge Dynamics. Proceedings of 9 th international conference on anaerobic digestion. Antwerpen pp 123$128,2001$.

[7] Korsak L., Cabezas A. and Moreno L. A Model of Kinetics and Mass Transfer in an $U A S B$ reactor. Proceedings of 4th international conference on environmental science and technology, 2008.

[8] Kalyuzhnyi S. Fedorovich V. and Lens P. Dispersed plug Flow Model for Upflow Anaerobic Sludge Bed Reactors with Focus on Granular Sludge Dynamics. Microbiol Biotecnol pp. 221-237, 2006.

[9] Bitton G. Wastewater Microbiology. 3rd Ed. Wiley Editor, 2005.

[10] García H., Rico C., García P. and Rico J. Flocculants Effect in Biomass Retention in a UASB Reactor Treating Dairy Manure. Bioresource Technology 99, 6028-6036, 2008.

[11] Sponza D. and Uluköy A. Kinetic of Carbonaceous Substrate in an Upflow Anaerobic Sludge Blanket (UASB) Reactor Treating 2,4 Dichlorophenol (2,4 DCP. Journal of Environmental Management 86, 121-131, (2008).

[12] Sponza D. and Işik M. Substrate Removal Kinetics in an Upflow Anaerobic Sludge Blanket Reactor Decolorising Simulated Textile Wastewater. Process Biochemistry 40, 1189-1198, 2005. 